\title{
A Kidney Exchange Clearinghouse in New England
}

\section{Citation}

Roth, Alvin E., Tayfun Sönmez , and M. Utku Ünver. 2005. A Kidney Exchange Clearinghouse in New England. American Economic Review 95, no. 2: 376-380.

\section{Published Version}

http://www.aeaweb.org/articles.php?doi=10.1257/000282805774669989;http://

dx.doi.org/10.1257/000282805774669989

\section{Permanent link}

http://nrs.harvard.edu/urn-3:HUL.InstRepos:2562810

\section{Terms of Use}

This article was downloaded from Harvard University's DASH repository, and is made available under the terms and conditions applicable to Other Posted Material, as set forth at http:// nrs.harvard.edu/urn-3:HUL.InstRepos:dash.current.terms-of-use\#LAA

\section{Share Your Story}

The Harvard community has made this article openly available.

Please share how this access benefits you. Submit a story.

Accessibility 


\title{
A Kidney Exchange Clearinghouse in New England
}

\author{
By Alvin E. Roth, Tayfun Sönmez, and M. UtKu ÜNveR*
}

In September, 2004, the Renal Transplant Oversight Committee of New England approved the establishment of a clearinghouse for kidney exchange, proposed by Francis Delmonico, Susan Saidman, and the three authors of this paper. We outline here the potential gains from kidney exchange and discuss practical constraints encountered as we begin designing and implementing a matching mechanism.

\section{Background}

In 2003 there were 8,665 transplants of deceased donor kidneys for the approximately 60,000 patients waiting for such transplants in the United States. While waiting, 3,436 patients died. There were also 6,464 kidney transplants from living donors (Scientific Registry of Transplant Recipients web site). ${ }^{1}$ Live donation is an option for kidneys, since healthy people have two and can remain healthy with one. While it is illegal to buy or sell organs, there have started to be kidney exchanges involving two donor-patient pairs such that each (living) donor cannot give a kidney to the intended recipient because of blood type or immunological incompatibility, but each patient can receive a kidney from the other donor. So far these have been rare: as of December 2004, only five exchanges had been performed in the 14 transplant centers in New England. One reason there have been so few kidney exchanges is that there have not been databases of incompatible patient-donor pairs. Incompatible donors were simply sent home. (Databases are now being assembled not only in New England, but also in Ohio and Baltimore.)

\footnotetext{
* Roth: Harvard Business School and Department of Economics, Harvard University, Cambridge, MA 02138 (e-mail: aroth@hbs.edu); Sönmez: Koç University, Istanbul, Turkey, and Harvard University (e-mail: tsonmez@ ku.edu.tr); Ünver: Koç University (e-mail: uunver@ku. edu.tr). We are grateful to Francis Delmonico and Susan Saidman for comments, and to NSF for support.

${ }^{1}\langle$ http://www.ustransplant.org/srtr.php〉
}

Lainie Friedman Ross et al. (1997) discussed the possibility of exchange between incompatible patient-donor pairs. Not only have a few such two-way exchanges been performed, but two three-way exchanges (in which the donor kidney from one pair is transplanted into the patient in a second pair, whose donor kidney goes to a third pair, whose donor kidney goes to the first pair) have been performed at Johns Hopkins. There have also been a number of "list exchanges" in which an incompatible patientdonor pair makes a donation to someone on the waiting list for a cadaver kidney, in return for the patient in the pair receiving high priority for a cadaver kidney when one becomes available.

\section{Scope and Design of a Kidney Clearinghouse:}

In Roth et al. (2004a), we considered how to organize all these kinds of exchanges efficiently, in a way that would give patients and their doctors straightforward incentives. (Because medical information is decentralized, some of the procedures for allocating cadaver organs have experienced incentive problems.) We modeled patients as having strict preferences over compatible kidneys, and we allowed exchanges among any number of patient-donor pairs (including not only incompatible pairs, but also compatible pairs who might nevertheless be able, through exchange, to obtain a preferred kidney). We allowed list exchanges to be integrated with live exchanges, so a patient-donor pair who decided to exchange their kidney for priority on the deceased donor list would not necessarily donate their kidney to someone on the list, but might instead donate their kidney to another patient-donor pair who would in turn donate a kidney to the list (or to another pair who would in turn donate a kidney to the list, etc.).

In our model each agent is a patient and her donor(s). Agents have strict preferences over other agents (based on compatibility, closeness 
of tissue match, and age of donor), and over priority on the cadaver wait-list.

If we exclude list exchange, this is the "housing market" of Lloyd Shapley and Herbert Scarf (1974), and David Gale's method of top trading cycles (TTC) produces efficient, core allocations. There is a unique such allocation (Roth and Andrew Postlewaite, 1977), and the mechanism that selects it is dominant-strategy incentive-compatible (Roth, 1982).

TTC works as follows: Each agent points to her most preferred agent (the patient with the agent's favorite donor). There is at least one cycle [an ordered list of agents $\left(a_{1}, a_{2}, \ldots, a_{n}\right)$ in which each agent points to the next, and agent $a_{n}$ points to $\left.a_{1}\right]$, and no agent can be part of more than one cycle. The implied exchange in each cycle is carried out, and the procedure continues with each remaining agent pointing to her favorite among the remaining agents.

When list exchange is included the model is close to the "room assignment" of Atila Abdulkadiroğlu and Sönmez (1999). At some point of the TTC procedure there may be no cycles, but only " $w$-chains" in which $a_{n}$ is pointing to the waiting list. An agent may be part of several $w$-chains and therefore the procedure needs a selection rule for $w$-chains. In Roth et al. (2004a) we called this class of procedures top trading cycles and chains (TTCC) and identified a version that is Pareto efficient and dominantstrategy incentive-compatible.

To solve one aspect of the incentive problem, all surgeries in a live-donor exchange are conducted simultaneously. Thus a two-way exchange (involving just two patient-donor pairs) involves four simultaneous surgeries, a threeway exchange involves six, and so on.

\section{Logistical Constraints:}

Our medical colleagues worried that, at least initially, they could not manage exchanges larger than two-way. They were also inclined to exclude list exchanges, and to allow only incompatible patient-donor pairs to participate. As a first approximation, their feeling was that a patient should be indifferent between any compatible exchanges.

Therefore, in Roth et al. (2004b), each agent is a patient with incompatible donors and is indifferent between all donors compatible with her. No exchange larger than two-way is feasible. Building on well-known results in graph theory we showed that there are constrainedefficient dominant-strategy incentive-compatible mechanisms. These include deterministic "priority" mechanisms like those organ banks use to allocate cadaver organs, and stochastic mechanisms that address equity considerations.

The gains from kidney exchange will depend on several factors including:

(i) the size of the patient-donor database;

(ii) whether list exchanges are included (while list exchanges have distributional implications for the deceased donor wait-list, their inclusion increases the potential gains from exchange);

(iii) the maximum number of transplants that can be simultaneously carried out (equivalently, the size of largest feasible cycle and/or $w$-chain); and

(iv) whether compatible patient-donor pairs can participate in exchange.

Consider pairs A and B: donor A is compatible with both patients, but donor B is compatible only with patient A. While donor A can directly give her kidney to patient $\mathrm{A}$, both patients receive a kidney if pairs $\mathrm{A}$ and $\mathrm{B}$ exchange. Such an exchange is called an altruistically unbalanced exchange (E. Steve Woodle and Ross, 1998) and is unlikely to be recommended to couple A as long as such exchanges are unusual. But if patients have strict preferences over donors, it could be that both pairs obtain a preferred kidney from such an exchange. (Consideration of compatible pairs, and altruistically unbalanced exchanges, will help us estimate an upper bound on the gains that can be achieved.)

We turn to simulations to estimate the impact of each of these factors on the number of patients who can benefit from exchange.

\section{Simulations}

For simplicity we consider non-blood-related patient-donor pairs. Distributions of blood types ( 48 percent $\mathrm{O}, 34$ percent $\mathrm{A}, 14$ percent $\mathrm{B}$, 
4 percent AB), PRA levels (discussed below), and gender of the patients (41 percent female), and percentage of spouses among the unrelated donors (49 percent) are from the UNOS/OPTN data. $^{2}$

Tissue-type incompatibility (a positive crossmatch) arises when a patient has antibodies against a donor protein. (The positive crossmatch probability between female patients and their husbands is approximately 33 percent, compared to approximately 11 percent between random pairs (Stefanos Zenios et al., 2000), because antibodies can develop during childbirth.) Patients in the UNOS database are divided into three groups based on the odds that they have a crossmatch with a random donor. For simplicity we simulate patients in discrete PRA (percent reactive antibody) levels:

(i) 70 percent low-PRA patients, each of whom has a positive crossmatch probability of 5 percent with a random donor,

(ii) 20 percent medium-PRA patients, each of whom has a positive crossmatch probability of 45 percent, and

(iii) 10 percent high-PRA patients, each of whom has a positive crossmatch probability of 90 percent.

We randomly simulate patient-donor pairs using Monte-Carlo simulation size of 100 random population constructions for each of the 16 scenarios described below:

(1) We consider two population sizes: 25 and 100.

(2) We consider including compatible pairs in exchange as well as excluding them. (For example, in a population of 25 patient-donor pairs, if compatible pairs are excluded from exchange, only the smaller number of incompatible pairs will be available for exchange, and these will have a different distribution of characteristics than the general

\footnotetext{
${ }^{2}$ UNOS/OPTN 2003 Annual Report, 1993-2002 〈http:// www.optn.org $\rangle$. Patient characteristics are from new waiting-list registrations, living donor relational type is from living-donor transplants data.
}

population; $\mathrm{O}$ donors will be rare, and high PRA patients will be more common.)

(3) Either

(a) list exchanges are unavailable; or

(b) list exchanges are available but only 40 percent of incompatible pairs consider a transplant from a deceased donor and only if a live donor is unavailable.

(4) The largest feasible cycle/w-chain is either 2 or unbounded.

These possibilities yield $2 \times 2 \times 2 \times 2=16$ scenarios, and for each realization we search for a feasible exchange that includes the maximum number of patients.

For simplicity we assume that patients are indifferent between compatible live donors but prefer any such donor to priority on the deceased-donor wait-list. We use versions of Jack Edmond's (1965) algorithm to find a maximal exchange when the largest feasible cycle/ $w$-chain is 2 . We know of no efficient algorithm to determine a maximal exchange when cycle/ $w$-chain size is unbounded. In these scenarios we search for a maximal exchange among efficient matchings via the TTCC algorithm.

Table 1 makes clear that the gains from all kinds of exchange increase as the population $n$ of patient-donor pairs grows. The exchanges that are initially likely to be achievable are those involving no list exchange ( 0 percent wait-list), and only incompatible patient-donor pairs. When only two-way exchanges are feasible, exchange yields on average an additional 3.96 such transplants when $n=25$ (16 percent of the patient population), but 23.04 when $n=100$. Allowing list exchange, or allowing larger than two-way exchanges each gives a comparable increase in the number of transplants that can be achieved.

The largest gains in the table come from including compatible pairs in the population eligible for exchange. As the bottom of Table 1 indicates, it is at least conceivable that in a large population in which all patient-donor pairs could participate in exchange, virtually every patient (98.83 percent) with a willing donor would be able to receive a kidney. But we emphasize that this is an upper bound, since for many compatible pairs, exchange will not be desirable. 
Table 1 -Simulation Results

\begin{tabular}{|c|c|c|c|c|c|}
\hline \multirow{2}{*}{$\begin{array}{l}\text { Comp. } \\
\text { pairs }\end{array}$} & \multirow[b]{2}{*}{$n$} & \multirow[b]{2}{*}{ WL } & \multicolumn{3}{|c|}{ Transplants } \\
\hline & & & Own & Ex. & $w$-List \\
\hline \multicolumn{6}{|c|}{ A. Two-Way Exchange: } \\
\hline \multirow[t]{4}{*}{ Out } & 25 & 0 & 11.56 & 3.96 & 0 \\
\hline & & 40 & 11.56 & 5.76 & 3.71 \\
\hline & 100 & 0 & 47.49 & 23.04 & 0 \\
\hline & & 40 & 47.49 & 28.79 & 11.48 \\
\hline \multirow[t]{4}{*}{ In } & 25 & 0 & 1.33 & 19.00 & 0 \\
\hline & & 40 & 1.33 & 19.63 & 2.12 \\
\hline & 100 & 0 & 1.01 & 90.14 & 0 \\
\hline & & 40 & 1.01 & 91.35 & 4.70 \\
\hline
\end{tabular}

B. Unrestricted Exchange:

\begin{tabular}{crrrrl} 
Out & 25 & 0 & 11.56 & 5.33 & 0 \\
& & 40 & 11.56 & 6.32 & 3.82 \\
& 100 & 0 & 47.49 & 28.71 & 0 \\
& & 40 & 47.49 & 30.38 & 11.82 \\
In & 25 & 0 & 1.44 & 20.30 & 0 \\
& & 40 & 1.50 & 20.29 & 2.20 \\
& 100 & 0 & 1.67 & 92.68 & 0 \\
& & 40 & 1.61 & 92.55 & 4.67 \\
\hline
\end{tabular}

Notes: The table reports the average number of patients receiving a transplant in each scenario (through own-donor, through an exchange, and through being sent to the top of the waiting list). Key to column headings: Comp. pairs = compatible pairs (excluded ["out"] or included ["in"]); $n=$ population size; $\mathrm{WL}=$ percentage of wait-list options; Own $=$ number of patients receiving own donor kidneys; Ex. = number of patients participating in an exchange; $w$-List $=$ number of patients who get priority in the waiting list through list exchange.

\section{Conclusions}

Kidney exchange is likely to proceed incrementally, starting with the simplest cases (twoway exchange) and the patients who can benefit most (incompatible pairs). Roth et al. (2005) show that most of the gain from larger than two-way exchange comes from three-way exchange, and so we are hopeful that it will be possible to achieve these gains in the near term. It may also be possible to include list exchanges and nondirected donors (altruistic living donors who do not specify a particular patient). Each of these increases in the scope of exchange will necessitate design changes in the clearinghouse, and there are open theoretical problems remaining for some of them (as is to be expected; cf. the examples in Roth [2002]).

It seems likely that, until exchange becomes well established, only incompatible patient- donor pairs will be included, as surgeons will be reluctant to advise compatible pairs not to proceed with their own transplant. However, as exchange becomes more routine, there will be opportunities for mutually beneficial exchange between, for example, a 25-year-old patient with a compatible 50-year-old donor and a 50-year-old patient with an incompatible 25-year-old donor.

Fortunately, the gains from even the simplest exchanges are large, and achievable.

\section{REFERENCES}

Abdulkadiroğlu, Atila and Sönmez, Tayfun. "House Allocation with Existing Tenants." Journal of Economic Theory, 1999, 88(2), pp. 233-60.

Edmonds, Jack. "Paths, Trees, Flowers." Canadian Journal of Mathematics, 1965, 17, pp. 449-67.

Ross, Lainie Friedman; Rubin, David T.; Siegler, Mark; Josephson, Michelle A.; Thistlethwaite, J. Richard, Jr. and Woodle, E. Steve. "Ethics of a Paired-Kidney-Exchange Program." New England Journal of Medicine, 1997, 336(24), pp. 1752-55.

Roth, Alvin E. "Incentive Compatibility in a Market with Indivisibilities." Economics Letters, 1982, 9(2), pp. 127-32.

Roth, Alvin E. "The Economist as Engineer: Game Theory, Experimental Economics, and Computation as Tools of Design Economics." Econometrica, 2002, 70(4), pp. 1341-78.

Roth, Alvin E. and Postlewaite, Andrew. "Weak versus Strong Domination in a Market with Indivisible Goods." Journal of Mathematical Economics, 1977, 4(2), pp. 131-37.

Roth, Alvin E.; Sönmez, Tayfun and Ünver, M. Utku. "Kidney Exchange." Quarterly Journal of Economics, 2004a, 119(2), pp. 457-88.

Roth, Alvin E.; Sönmez, Tayfun and Ünver, M. Utku. "Pairwise Kidney Exchange." National Bureau of Economic Research (Cambridge, MA) Working Paper No. 10698, 2004b; Journal of Economic Theory (forthcoming).

Roth, Alvin E.; Sönmez, Tayfun and Ünver, M. Utku. "Efficient Kidney Exchange: Coincidence of Wants in a Structured Market." National Bureau of Economic Research 
(Cambridge, MA) Working Paper No. 11402, 2005.

Shapley, Lloyd and Scarf, Herbert. "On Cores and Indivisibility." Journal of Mathematical Economics, 1974, 1(1), pp. 23-28.

Woodle, E. Steve and Ross, Lainie Friedman. "Paired Exchanges Should Be Part of the Solution to ABO Incompatibility in Living
Donor Kidney Transplantation." Transplantation, 1998, 66(3), pp. 406-7.

Zenios, Stefanos; Woodle, E. Steve and Ross, Lainie Friedman. "Primum Non Nocere: Avoiding Harm to Vulnerable Wait List Candidates in an Indirect Kidney Exchange." Transplantation, 2001, 72(4), pp. 648-54. 